\title{
New spectral criteria for almost periodic solutions of evolution equations
}

\author{
by \\ Toshiki Naito (Tokyo), Nguren Van Minh (Hanoi and Tokyo), \\ and Jong Son Shin (Tokyo)
}

\begin{abstract}
We present a general spectral decomposition technique for bounded solutions to inhomogeneous linear periodic evolution equations of the form $\dot{x}=A(t) x+f(t)(*)$, with $f$ having precompact range, which is then applied to find new spectral criteria for the existence of almost periodic solutions with specific spectral properties in the resonant case where $\overline{e^{i \mathrm{sp}(f)}}$ may intersect the spectrum of the monodromy operator $P$ of $(*)$ (here $\operatorname{sp}(f)$ denotes the Carleman spectrum of $f)$. We show that if $(*)$ has a bounded uniformly continuous mild solution $u$ and $\sigma_{\Gamma}(P) \backslash \overline{e^{i \operatorname{sp}(f)}}$ is closed, where $\sigma_{\Gamma}(P)$ denotes the part of $\sigma(P)$ on the unit circle, then $(*)$ has a bounded uniformly continuous mild solution $w$ such that $\overline{e^{i \mathrm{sp}(w)}}=\overline{e^{i \mathrm{sp}(f)}}$. Moreover, $w$ is a "spectral component" of $u$. This allows us to solve the general Massera-type problem for almost periodic solutions. Various spectral criteria for the existence of almost periodic and quasi-periodic mild solutions to $(*)$ are given.
\end{abstract}

1. Introduction. We consider the following linear inhomogeneous integral equation:

$$
x(t)=U(t, s) x(s)+\int_{s}^{t} U(t, \xi) g(\xi) d \xi, \quad \forall t \geq s ; t, s \in \mathbb{R},
$$

where $f$ is continuous, $x(t) \in \mathbf{X}, \mathbf{X}$ is a Banach space, and $(U(t, s))_{t \geq s}$ is assumed to be a 1-periodic evolutionary process on $\mathbf{X}$ (see Definition 2.2 below). In turn, this notion of evolutionary processes arises naturally from

2000 Mathematics Subject Classification: Primary 34G10, 47D06; Secondary 34C27, 35B15, 45M15.

Key words and phrases: periodic evolution equation, spectrum of functions, spectral decomposition, almost periodic mild solution, quasi-periodic mild solution, Fourier coefficient.

The main results of this paper were announced by the second author (N.V.M.) at the RIMS workshop, Kyoto University, November 10-13, 1998 and the First International Conference on Semigroups: Theory and Applications, Newport Beach (California, U.S.A.), December 14-18, 1998. 
the well-posed evolution equations

$$
\frac{d x}{d t}=A(t) x+f(t), \quad t \in \mathbb{R}, x \in \mathbf{X},
$$

where $A(t)$ is a linear (in general, unbounded) operator for every fixed $t$ and is 1-periodic in $t$ (see e.g. [Paz]).

Recently, there is an increasing interest in investigating the asymptotic behavior of solutions to the well-posed equation (2) by means of (1) (see e.g. $[\mathrm{Na}],[\mathrm{Ne}])$. An interesting problem in the qualitative theory of solutions to (1) is to find conditions for the existence of (almost) periodic solutions. In this direction, it is known (see e.g. [Pr], $[\mathrm{V}-\mathrm{S}]$ for the autonomous case and $[\mathrm{N}-\mathrm{M}],[\mathrm{M}-\mathrm{N}-\mathrm{M}]$ and also $[\mathrm{B}-\mathrm{H}-\mathrm{R}]$ for the periodic case) that if the following nonresonant condition holds:

$$
\left(\sigma(P) \cap S^{1}\right) \cap \overline{e^{i \operatorname{sp}(f)}}=\emptyset,
$$

where $P:=U(1,0), S^{1}$ denotes the unit circle of the complex plane, $f$ is almost periodic, $\operatorname{sp}(f)$ is the Carleman spectrum of $f$ whose definition is given in the next section and $\overline{e^{i \operatorname{sp}(f)}}$ is the closure of the set $\left\{e^{i \xi}: \xi \in \operatorname{sp}(f)\right\}$ in the usual topology of the complex plane, then there exists an almost periodic solution $x_{f}$ to (1) which is unique if one requires

$$
\overline{e^{i \operatorname{sp}\left(x_{f}\right)}} \subset \overline{e^{i \operatorname{sp}(f)}} \text {. }
$$

We may ask what happens in the resonant case where condition (3) fails. In fact, in the particular case where the forcing term $f$ is 1-periodic and the monodromy operator $P$ is compact this question has been answered under the additional assumption that there exists a bounded uniformly continuous solution to (1). Historically, this question goes back to a classical result by Massera [Ma] saying that in the finite-dimensional case, for (2) to have a 1-periodic solution it is necessary and sufficient that it has a bounded solution (see [C-H], [D-M, Thm. 11.20], [S-N], [N-M-M-S] for extensions to the infinite-dimensional case, and also [F, Example 12.5], [J], [S-Y], [Ya] for related results and counterexamples for almost periodic equations).

It is the purpose of this paper to give an answer to a general problem as the one mentioned above (Massera-type problem): Let (1) have a bounded (uniformly continuous) solution $x_{f}$ with given almost periodic forcing term $f$. When does (1) have an almost periodic solution $w$ (which may be different from $x_{f}$ ) such that

$$
\overline{e^{i \operatorname{sp}(w)}} \subset \overline{e^{i \operatorname{sp}(f)}} ?
$$

In connection with this problem we note that various conditions have been found on the bounded solution and the countability of the part $\sigma(P) \cap$ $S^{1}$ of the spectrum so that the bounded solution itself is almost periodic, or more generally, together with $f$ belongs to a given function space $\mathcal{F}$ (see e.g. 
$[\mathrm{L}-\mathrm{Z}],[\mathrm{A}-\mathrm{B}],[\mathrm{R}-\mathrm{V}],[\mathrm{Ba}], \ldots)$. Here we note that this philosophy, in general, does not apply to the Massera-type problem for almost periodic solutions. In fact, it is not difficult to give an example in which $f$ is 1-periodic and a bounded (uniformly continuous) solution to (1) exists, but this bounded solution itself is not 1-periodic.

Our method is to employ the evolution semigroup (see Definition 2.3 below) associated with $(U(t, s))_{t \geq s}$ to study the harmonic analysis of bounded solutions to (1). As a result we prove a spectral decomposition theorem for bounded solutions (Theorems 3.3 and 3.5) which seems to be useful in dealing with the above Massera-type problem. In fact, we apply the spectral decomposition theorem to find new spectral criteria for the existence of almost periodic solutions, and consider particular cases to show the usefulness of this technique. More concretely, even in the case where condition (3) fails we can still prove the existence of a bounded uniformly continuous solution $w$ to (1) such that $\overline{e^{i \operatorname{sp}(w)}}=\overline{e^{i \operatorname{sp}(f)}}$ provided that $\left(\sigma(P) \cap S^{1}\right) \backslash \overline{e^{i \operatorname{sp}(f)}}$ is closed, and that (1) has a bounded uniformly continuous solution $u$ (Corollary 4.2). Since $w$ is a "spectral component" of $u$, in case $u$ is almost periodic the Fourier series of $w$ is part of that of $u$ (Corollary 4.3). Corollary 4.4 deals with a particular autonomous case in which Corollary 4.2 fails to give a spectral criterion for the existence of quasi-periodic mild solutions.

2. Preliminaries. Throughout the paper we use the following notations: $\mathbb{N}, \mathbb{Z}, \mathbb{R}, \mathbb{C}$ stand for the sets of natural, integer, real, and complex numbers, respectively; $S^{1}$ denotes the unit circle in the complex plane $\mathbb{C}$; $\mathbf{X}$ denotes a given complex Banach space. If $T$ is a linear operator on $\mathbf{X}$, then $D(T)$ stands for its domain. Given two Banach spaces $\mathbf{X}, \mathbf{Y}$ we denote by $L(\mathbf{X}, \mathbf{Y})$ the space of all bounded linear operators from $\mathbf{X}$ to $\mathbf{Y}$. As usual, $\sigma(T), \varrho(T), R(\lambda, T)$ are the notations for the spectrum, resolvent set and resolvent of the operator $T$. The notations $\mathrm{BC}(\mathbb{R}, \mathbf{X}), \operatorname{BUC}(\mathbb{R}, \mathbf{X}), \operatorname{AP}(\mathbf{X})$ stand for the spaces of all $\mathbf{X}$-valued bounded continuous functions, bounded uniformly continuous functions on $\mathbb{R}$ and the subspace of almost periodic (in Bohr's sense) functions, respectively. Recall that a function $f \in \mathrm{BUC}(\mathbb{R}, \mathbf{X})$ is called almost periodic (in Bohr's sense) if $\{S(\tau) f: \tau \in \mathbb{R}\}$ is relatively compact in $\operatorname{BUC}(\mathbb{R}, \mathbf{X})$, where $(S(t))_{t \in \mathbb{R}}$ is the group of translations on $\mathrm{BUC}(\mathbb{R}, \mathbf{X})$. It is known (see e.g. [L-Z]) that the set of Fourier-Bohr exponents of an almost periodic function $f$ is defined to be the set of all nonzero real numbers of the form

$$
\alpha=\lim _{N \rightarrow \infty} \frac{1}{2 N} \int_{-N}^{N} e^{-i \varrho t} f(t) d t, \quad \varrho \in \mathbb{R} .
$$

The set of Fourier-Bohr exponents of an almost periodic function $f$ is at most countable and will be denoted by $\exp (f)$. 
We use the notion of the Carleman spectrum of a bounded continuous function $u$ on the real line, denoted by $\operatorname{sp}(u)$, consisting of $\xi \in \mathbb{R}$ such that the Fourier-Carleman transform of $u$,

$$
\widehat{u}(\lambda)= \begin{cases}\int_{0}^{\infty} e^{-\lambda t} u(t) d t & (\operatorname{Re} \lambda>0), \\ -\int_{0}^{\infty} e^{\lambda t} u(-t) d t & (\operatorname{Re} \lambda<0),\end{cases}
$$

has no holomorphic extension to a neighborhood of $i \xi$ (see e.g. [Pr, pp. 19-27]). We refer the reader to [D], [Ka], [Pr], [A-B] for more information on this notion as well as its relations to other notions of spectrum. Basic properties of the Carleman spectrum of a function and its relation to the behavior of the function are listed below for the reader's convenience.

Proposition 2.1. Let $f, g_{n} \in \mathrm{BUC}(\mathbb{R}, \mathbf{X})$ be such that $\lim _{n \rightarrow \infty}\left\|g_{n}-f\right\|$ $=0$. Then:

(i) $\operatorname{sp}(f)$ is closed.

(ii) $\operatorname{sp}(f(\cdot+h))=\operatorname{sp}(f)$.

(iii) If $\alpha \in \mathbb{C} \backslash\{0\}$, then $\operatorname{sp}(\alpha f)=\operatorname{sp}(f)$.

(iv) If $\operatorname{sp}\left(g_{n}\right) \subset \Lambda$ for all $n \in \mathbb{N}$, then $\operatorname{sp}(f) \subset \bar{\Lambda}$.

(v) $\operatorname{sp}(\psi+f) \subset \operatorname{sp}(f) \cup \operatorname{sp}(\psi)$ for all $\psi \in \mathrm{BC}(\mathbb{R}, \mathbf{X})$.

(vi) If $u$ is uniformly continuous, $\operatorname{sp}(u)$ is countable and $\mathbf{X}$ does not contain any subspace which is isomorphic to the sequence space $c_{0}$, then $u$ is almost periodic.

(vii) If $u$ is uniformly continuous and $\operatorname{sp}(u)$ is discrete, then $u$ is almost periodic.

Proof. For the proof we refer the reader to [Pr, Proposition 0.4, p. 20, Theorem 0.8, p. 21] and [A-S], [L-Z, Chap. 6].

For simplicity we use the following notation throughout: $\sigma(g):=\overline{e^{i \operatorname{sp}(g)}}$ for every bounded uniformly continuous function $g$, and $\sigma_{\Gamma}(P)=\sigma(P) \cap S^{1}$. If $f$ is almost periodic, then $\operatorname{sp}(f)=\overline{\exp (f)}$.

The following notion will be used throughout the paper:

Definition 2.2. A family $(U(t, s))_{t \geq s}(t, s \in \mathbb{R})$ of bounded linear operators from a Banach space $\mathbf{X}$ to itself is called a 1-periodic strongly continuous evolutionary process if:

(i) $U(t, t)=I$ for all $t \in \mathbb{R}$,

(ii) $U(t, s) U(s, r)=U(t, r)$ for all $t \geq s \geq r$,

(iii) the map $(t, s) \mapsto U(t, s) x$ is continuous for every fixed $x \in \mathbf{X}$,

(iv) $U(t+1, s+1)=U(t, s)$ for all $t \geq s$,

(v) $\|U(t, s)\|<N e^{\omega(t-s)}$ for some positive $N$, $\omega$ independent of $t \geq s$.

The operator $U(1,0)$ is called the monodromy operator of the evolutionary process $(U(t, s))_{t \geq s}$ and denoted by $P$ throughout this paper. Note that 
in this paper the period of evolutionary processes is assumed to be 1 merely for simplicity.

Definition 2.3. Let $(U(t, s))_{t>s}(t, s \in \mathbb{R})$ be a 1-periodic strongly continuous evolutionary process and $\mathbf{F}$ be a closed subspace of BUC $(\mathbb{R}, \mathbf{X})$ such that for every fixed $h>0$ and $g \in \mathbf{F}$ the map $t \mapsto U(t, t-h) g(t-h)$ belongs to $\mathbf{F}$. Then the semigroup $\left(T^{h}\right)_{h \geq 0}$ of operators on $\mathbf{F}$, defined by the formula

$$
T^{h} g(t)=U(t, t-h) g(t-h), \quad \forall t \in \mathbb{R}, h \geq 0, g \in \mathbf{F},
$$

is called the evolution semigroup associated with the process $(U(t, s))_{t \geq s}$.

We refer the reader to the papers $[\mathrm{A}-\mathrm{M}],[\mathrm{N}-\mathrm{M}],[\mathrm{M}-\mathrm{N}-\mathrm{M}]$ for more information on the applications of this notion to the study of the existence of bounded and almost periodic solutions as well as the references therein for information on other applications to the study of exponential dichotomy and stability of solutions to (1).

In the case where the evolution semigroup $\left(T^{h}\right)_{h \geq 0}$ is strongly continuous on $\mathbf{F}$, [A-M, Thm. 2] yields an explicit formula for the generator $\mathcal{A}$ of $\left(T^{h}\right)_{h \geq 0}$. In fact, similarly to [N-M, Lemma 2] we have the following:

LEMMA 2.4. Let $\left(T^{h}\right)_{h \geq 0}$ be strongly continuous on $\mathbf{F}$, a closed subspace of $\mathrm{BUC}(\mathbb{R}, \mathbf{X})$. Then its generator $\mathcal{A}$ is the operator on $\mathbf{F}$ with $D(\mathcal{A})$ consisting of all $g \in \mathbf{F}$ such that $g$ is a solution to (1) for some $f \in \mathbf{F}$ (in this case such a function $f$ is unique), and by definition, $\mathcal{A} g=-f$.

It is not hard to see that in the finite-dimensional case in which $(U(t, s))_{t \geq s}$ is the Cauchy operator associated with the ordinary differential equation

$$
\frac{d x}{d t}=A(t) x, \quad x \in \mathbb{R}^{n}, t \in \mathbb{R},
$$

where $A(t)$ is a continuous $n \times n$-matrix-valued function, the infinitesimal generator $\mathcal{A}$ of the semigroup $\left(T^{h}\right)_{h \geq 0}$ is nothing but $(\mathcal{A} g)(t)=A(t) g(t)-$ $d g(t) / d t$. However, in general, it is not the case. In fact, the operator $\mathcal{A}$ is the closure of the operator $A(t)-d / d t$ in a suitable topology (see [M-N-M] for the autonomous case and its references for more information).

3. Spectral decomposition. Consider the subspace $\mathcal{M} \subset \mathrm{BUC}(\mathbb{R}, \mathbf{X})$ consisting of all functions $v \in \mathrm{BUC}(\mathbb{R}, \mathbf{X})$ such that

$$
\overline{e^{i \operatorname{sp}(v)}}:=\sigma(v) \subset S_{1} \cup S_{2},
$$

where $S_{1}, S_{2} \subset S^{1}$ are disjoint closed subsets of the unit circle. We set $\mathcal{M}_{v}=\overline{\operatorname{span}\{S(t) v: t \in \mathbb{R}\}}$ (the closure of the linear subspace of $\mathrm{BUC}(\mathbb{R}, \mathbf{X})$ spanned by $\{S(t) v: t \in \mathbb{R}\})$, where $(S(t))_{t \in \mathbb{R}}$ is the translation group on $\operatorname{BUC}(\mathbb{R}, \mathbf{X})$, i.e. $S(t) v(s)=v(t+s)$ for $t, s \in \mathbb{R}$. 
THEOREM 3.1. Under the above notations and assumptions the function space $\mathcal{M}$ can be split into a direct sum $\mathcal{M}=\mathcal{M}_{1} \oplus \mathcal{M}_{2}$ such that $v \in \mathcal{M}_{i}$ if and only if $\sigma(v) \subset S_{i}$ for $i=1,2$.

Proof. Denote by $\Lambda_{i} \subset \operatorname{BUC}(\mathbb{R}, \mathbf{X})$ the set of functions $u$ such that $\sigma(u) \subset S_{i}$ for $i=1,2$. Then obviously, $\Lambda_{i} \subset \mathcal{M}$. Moreover, they are closed linear subspaces of $\mathcal{M}, \Lambda_{1} \cap \Lambda_{2}=\{0\}$. We want to prove that

$$
\mathcal{M}=\Lambda_{1} \oplus \Lambda_{2} \text {. }
$$

To this end, it is sufficient to show that for any element $v \in \mathcal{M}$ we have $v=v_{1}+v_{2}$, where $v_{1} \in \Lambda_{1}, v_{2} \in \Lambda_{2}$. As is known (see e.g. [A-B])

$$
i \operatorname{sp}(v)=\sigma\left(\mathcal{D}_{\mathcal{M}_{v}}\right)
$$

where $\mathcal{D}_{\mathcal{M}_{v}}$ is the infinitesimal generator of the translation group $(S(t))_{t \in \mathbb{R}}$ on $\mathcal{M}_{v}$. Thus, by the Weak Spectral Mapping Theorem (see e.g. [E-Na], $[\mathrm{Na}])$

$$
\sigma\left(\left.S(1)\right|_{\mathcal{M}_{v}}\right)=\overline{e^{\sigma\left(\mathcal{D}_{\left.\mathcal{M}_{v}\right)}\right.}}=\sigma(v) \subset S_{1} \cup S_{2}
$$

Hence, there is a spectral projection in $\mathcal{M}_{v}$,

$$
P_{v}^{1}:=\frac{1}{2 i \pi} \int_{\gamma} R\left(\lambda,\left.S(1)\right|_{\mathcal{M}_{v}}\right) d \lambda,
$$

where $\gamma$ is a contour enclosing $S_{1}$ and disjoint from $S_{2}$ (or in general a union of finitely many such countours). This implies

$$
\sigma\left(\left.S(1)\right|_{\operatorname{Im} P_{v}^{1}}\right) \subset S_{1}, \quad \sigma\left(\left.S(1)\right|_{\operatorname{Ker} P_{v}^{1}}\right) \subset S_{2} .
$$

Now we show that $v=v_{1}+v_{2}$, where $v_{1}:=P_{v}^{1} v \in \Lambda_{1}$ and $v-v_{1}=: v_{2} \in \Lambda_{2}$. To this end, we will prove

$$
\sigma\left(v_{j}\right) \subset S_{j}, \quad \forall j=1,2 .
$$

In fact, we show that $\mathcal{M}_{v_{1}}=\operatorname{Im} P_{v}^{1}$. Obviously, in view of the invariance of $\operatorname{Im} P_{v}^{1}$ under translations we have $\mathcal{M}_{v_{1}} \subset \operatorname{Im} P_{v}^{1}$. We now show the converse. To this end, let $y \in \operatorname{Im} P_{v}^{1} \subset \mathcal{M}_{v}$. Then, by definition, there is a sequence $\left\{x_{n}\right\}_{n \in \mathbb{N}} \subset \operatorname{span}\{S(t) v: t \in \mathbb{R}\}$ such that $y=\lim _{n \rightarrow \infty} x_{n}$. Hence, $x_{n}$ can be represented in the form

$$
x_{n}=\sum_{k=1}^{N(n)} \alpha_{k, n} S\left(t_{k, n}\right) v, \quad \alpha_{k, n} \in \mathbb{C}, t_{k, n} \in \mathbb{R} \forall n .
$$

Since $y \in \operatorname{Im} P_{v}^{1} \subset \mathcal{M}_{v}$, we have $P_{v}^{1} y=y$. So, since $x_{n} \in \mathcal{M}_{v}$,

$$
y=P_{v}^{1} y=\lim _{n \rightarrow \infty} \sum_{k=1}^{N(n)} \alpha_{k, n} S\left(t_{k, n}\right) P_{v}^{1} v=\lim _{n \rightarrow \infty} \sum_{k=1}^{N(n)} \alpha_{k, n} S\left(t_{k, n}\right) v_{1} .
$$


This shows that $y \in \mathcal{M}_{v_{1}}$. Thus, by the Weak Spectral Mapping Theorem and (8),

$$
\overline{e^{i \operatorname{sp}\left(v_{1}\right)}}=\sigma\left(\left.S(1)\right|_{\mathcal{M}_{v_{1}}}\right)=\sigma\left(\left.S(1)\right|_{\operatorname{Im} P_{v}^{1}}\right) \subset S_{1} .
$$

By definition, $v_{1} \in \Lambda_{1}$, and similarly $v_{2} \in \Lambda_{2}$. Thus the theorem is proved.

Remark. Below for every $v \in \mathcal{M}$ we will call the functions $v_{j}, j=1,2$, as defined in the proof of Theorem 3.1, the spectral components of $v$. It is easily seen that if in the proof of Theorem 3.1, $v$ is assumed to be almost periodic, then so are both $v_{j}$.

Lemma 3.2. Let $u$ be a bounded uniformly continuous solution to (1). Then:

(i) We have

$$
\sigma(u) \subset \sigma_{\Gamma}(P) \cup \sigma(f) .
$$

(ii) If furthermore we assume that $f$ has precompact range, then

$$
\sigma(u) \supset \sigma(f) .
$$

Proof. (i) For the proof see [B-H-R, Proposition 3.2].

(ii) Under the assumptions it may be seen that the evolution semigroup $\left(T^{h}\right)_{h \geq 0}$ associated with $(U(t, s))_{t \geq s}$ is strongly continuous at $u \in$ $\mathrm{BUC}(\mathbb{R}, \mathbf{X})$ (this can be checked directly using (1)), and at $f$ (see [N-M, Lemma 2]). Hence, by Lemma 2.4,

$$
\lim _{h \rightarrow 0^{+}} \frac{T^{h} u-u}{h}=\mathcal{A} u=-f .
$$

Hence, to prove (12) it suffices to show that $\sigma\left(T^{h} u\right) \subset \sigma(u)$. In turn, this inclusion can be shown by using the definition of the spectrum of a function as in [V, Lemma 4.3], [B-H-R, Lemma 3.6].

REMARK. We refer the reader to $[\mathrm{L}-\mathrm{Z}],[\mathrm{Ba}],[\mathrm{A}-\mathrm{B}], \ldots$ for results related to the inclusion (11). The inclusion (12) seems to be new and yields a necessary condition for the existence of bounded solutions to (1).

We are now in a position to state the main result of this paper.

Theorem 3.3 (Spectral Decomposition Theorem). Let $u$ be a bounded uniformly continuous solution to (1). Moreover, let $f$ have precompact range and the sets $\sigma(f)$ and $\sigma(P) \cap S^{1}$ be contained in a disjoint union of closed subsets $S_{1}, \ldots, S_{k}$ of the unit circle. Then the solution $u$ can be decomposed into a sum of $k$ spectral components $u_{j}, j=1, \ldots, k$, such that each $u_{j}, j=$ $1, \ldots, k$, is a solution to (1) with $f=f_{j}, j=1, \ldots, k$, respectively, where $f=$ $\sum_{j=1}^{k} f_{j}$ is the decomposition of $f$ into the sum of spectral components as described in Theorem 3.1, i.e. $u=\sum_{j=1}^{k} u_{j}, \sigma\left(u_{j}\right), \sigma\left(f_{j}\right) \subset S_{j}, j=1, \ldots, k$, and $u_{j} \in \mathrm{BUC}(\mathbb{R}, \mathbf{X})$ is a solution to (1) with $f:=f_{j}$ for $j=1, \ldots, k$. 
Proof. Denote by $\mathcal{N}$ the subspace of $\mathrm{BUC}(\mathbb{R}, \mathbf{X})$ consisting of all functions $u$ such that $\sigma(u) \subset \bigcup_{j=1}^{k} S_{j}$. Then, by assumptions and Theorem 3.1 there are spectral projections $P_{1}, \ldots, P_{k}$ on $\mathcal{N}$ such that:

(i) $P_{j} P_{n}=0$ if $j \neq n$,

(ii) $\sum_{j=1}^{k} P_{j}=I$,

(iii) if $u \in \mathcal{N}$, then $\sigma\left(P_{j} u\right) \subset S_{j}$ for all $j=1, \ldots, k$.

Note that by Lemma 3.2 for every positive $h$ and $j=1, \ldots, k$ the operator $T^{h}$ leaves $\operatorname{Im} P_{j}$ invariant. Hence, $\mathcal{N}$ and $\operatorname{Im} P_{1}, \ldots, \operatorname{Im} P_{k}$ are invariant under the semigroup $\left(T^{h}\right)_{h \geq 0}$. Consequently, since $u$ is a solution to (1) and $f$ has precompact range, the evolution semigroup $\left(T^{h}\right)_{h \geq 0}$ is strongly continuous at $u$ and at $f$. Using the explicit formula for the generator of $\left(T^{h}\right)_{h \geq 0}$ as described in Lemma 2.4 we have

$$
\begin{aligned}
P_{j} f & =P_{j} \lim _{h \rightarrow 0^{+}} \frac{T^{h} u-u}{h}=P_{j} \lim _{h \rightarrow 0^{+}} \sum_{n=1}^{k} P_{n} \frac{T^{h} u-u}{h} \\
& =\lim _{h \rightarrow 0^{+}} \frac{T^{h} P_{j} u-P_{j} u}{h} .
\end{aligned}
$$

This yields that $P_{j} u$ is a solution to (1) with $f_{j}=P_{j} f$.

REMARK. If in Theorem 3.3 we assume furthermore that $f$ and $u$ are both almost periodic, then so are the spectral components $u_{j}, j=1, \ldots, k$.

Now we focus our attention on the autonomous equations of the form

$$
d x / d t=A x+f(t)
$$

where $A$ is the generator of a $C_{0}$-semigroup $(T(t))_{t>0}$, and $f \in \operatorname{BUC}(\mathbb{R}, \mathbf{X})$ has precompact range. We set $\sigma_{i}(A)=\{\lambda \in \mathbb{R}: i \lambda \in \sigma(A) \cap i \mathbb{R}\}$. By mild solutions of (15) we understand (see [Paz]) solutions to (1) with $U(t, s):=$ $T(t-s)$ for all $t \geq s$. As shown below, in this case we can refine the spectral decomposition technique to get stronger assertions whose usefulness will be shown in the next section where we deal with quasi-periodic solutions. To this end, we now prove the following lemma.

Lemma 3.4. Let (15) satisfy the above conditions, i.e. A generates a $C_{0}$-semigroup and $f \in \mathrm{BUC}(\mathbb{R}, \mathbf{X})$ has precompact range. Moreover, let $u$ be a bounded uniformly continuous mild solution to (15). Then

$$
\begin{aligned}
& \operatorname{sp}(u) \subset \sigma_{i}(A) \cup \operatorname{sp}(f), \\
& \operatorname{sp}(u) \supset \operatorname{sp}(f) .
\end{aligned}
$$

Proof. For (16) we can use the standard way of computing the Carleman transform of $u$ as done in [A-B, p. 373]. Then there is no difficulty to get (16) if one uses the definition of the spectrum of a function. 
To prove (17), note that $\operatorname{sp}\left(T^{h} u\right) \subset \operatorname{sp}(u)$ by the definition of $T^{h}$. Thus, using the argument of the proof of Lemma 3.2(ii) we have

$$
\operatorname{sp}(f)=\operatorname{sp}(-f)=\operatorname{sp}\left(\lim _{h \rightarrow 0^{+}} \frac{T^{h} u-u}{h}\right) \subset \operatorname{sp}(u) .
$$

The main result for the autonomous case is the following theorem.

Theorem 3.5. Let $A$ generate a $C_{0}$-semigroup and $f \in \mathrm{BUC}(\mathbb{R}, \mathbf{X})$ have precompact range. Moreover, let $u$ be a bounded uniformly continuous mild solution to (15).

(i) If $\overline{e^{i \sigma_{i}(A)}} \backslash \sigma(f)$ is closed, (15) has a bounded uniformly continuous mild solution $w$ such that $\sigma(w)=\sigma(f)$.

(ii) If $\sigma_{i}(A)$ is bounded and $\sigma_{i}(A) \backslash \mathrm{sp}(f)$ is closed, then (15) has a bounded uniformly continuous mild solution $w$ such that $\operatorname{sp}(w)=\operatorname{sp}(f)$.

Proof. (i) Apply the proof of Theorem 3.3 together with (16).

(ii) Under the assumptions there exists a continuous function $\psi$ which belongs to the Schwartz space of all $C^{\infty}$-functions on $\mathbb{R}$ with all derivatives decaying faster than any polynomial such that its Fourier transform $\widetilde{\psi}$ has $\sigma_{i}(A) \backslash \operatorname{sp}(f)$ as its support (which is compact in view of the assumptions). Hence, every bounded uniformly continuous function $g$ such that $\operatorname{sp}(g) \subset$ $\sigma_{i}(A) \cup \operatorname{sp}(f)$ can be decomposed into the sum of two spectral components as follows:

$$
g=g_{1}+g_{2}=\psi * g+(g-\psi * g),
$$

where $g_{1}=\psi * g$ and $g_{2}=g-\psi * g$. Moreover, this decomposition is continuous in the following sense: If $g^{(n)}, n=1,2, \ldots$, is a sequence in $\operatorname{BUC}(\mathbb{R}, \mathbf{X})$ with $\operatorname{sp}\left(g^{(n))} \subset \sigma_{i}(A) \cup \operatorname{sp}(f)\right.$ such that $\lim _{n} g^{(n)}=g$ in $\operatorname{BUC}(\mathbb{R}, \mathbf{X})$, then $\lim _{n} g_{1}^{(n)}=g_{1}$ and $\lim _{n} g_{2}^{(n)}=g_{2}$. Hence we have in fact proved a version of Theorem 3.1 which allows us to employ the proof of Theorem 3.3 to show (ii).

Remarks. (i) In view of the failure of the Spectral Mapping Theorem for general $C_{0}$-semigroups the condition in (i) is a little more general than that formulated in terms of $\sigma(T(1))$.

(ii) If we know beforehand that $u$ is almost periodic, then in the statement of Theorem 3.5 we can claim that the spectral component $w$ is almost periodic.

4. Spectral criteria for almost periodic solutions. This section will be devoted to some applications of the spectral decomposition theorem to proving the existence of almost periodic solutions with specific spectral properties. In particular, we will revisit the classical result by Massera on 
the existence of periodic solutions as well as its extensions. To this end, the following notion will play a key role.

Definition 4.1. Let $\sigma(f)$ and $\sigma_{\Gamma}(P)$ be defined as above. We say that the sets $\sigma(f)$ and $\sigma_{\Gamma}(P)$ satisfy the spectral separation condition if the set $\sigma_{\Gamma}(P) \backslash \sigma(f)$ is closed.

COROLlary 4.2. Let $f$ be almost periodic, and suppose $\sigma(f)$ and $\sigma_{\Gamma}(P)$ satisfy the spectral separation condition. Moreover, assume that $\sigma(f)$ is countable and $\mathbf{X}$ does not contain any subspace isomorphic to $c_{0}$. If there exists a bounded uniformly continuous solution $u$ to (1), then there exists an almost periodic solution $w$ to $(1)$ such that $\sigma(w)=\sigma(f)$.

Proof. We define $S_{1}:=\sigma(f)$ and $S_{2}:=\sigma_{\Gamma}(P) \backslash \sigma(f)$. Then, by Theorem 3.3, there exists a solution $w$ to (1) such that $\sigma(w) \subset \sigma(f)$. Using (12) we have $\sigma(w)=\sigma(f)$. In particular, by Proposition 2.1(vi), $w$ is almost periodic.

REMARKs. (i) If $\sigma(f)$ is finite, then by Proposition 2.1(vii) the condition that $\mathbf{X}$ does not contain any subspace isomorphic to $c_{0}$ can be dropped.

(ii) In the case where $\sigma_{\Gamma}(P)$ is countable it is known that with additional ergodic conditions on $u$ the solution $u$ has "similar spectral properties" to $f$ (see e.g. [L-Z], $[\mathrm{Ba}],[\mathrm{A}-\mathrm{B}],[\mathrm{B}-\mathrm{H}-\mathrm{R}], \ldots$ ). However, in many cases it is not expected that the solution $u$ itself has similar spectral properties to $f$ as in the Massera-type problem (see [Ma], [C-H], [S-N], [N-M-M-S],...).

(iii) In the case where $P$ is compact (or merely $\sigma_{\Gamma}(P)$ is finite) the spectral separation condition is always satisfied. Hence, we have a natural extension of Massera's result to almost periodic solutions. In this case see also Corollary 4.3 below.

(iv) We emphasize that the solution $w$ in the statement of Corollary 4.2 is a " $\sigma(f)$-spectral component" of the bounded solution $u$. This will be helpful in finding the Fourier coefficients of $w$ as part of those of $u$.

(v) In view of the inclusion (12), $w$ may be seen as a "minimal" solution in some sense.

Corollary 4.3. Let all assumptions of Corollary 4.2 be satisfied. Moreover, let $\sigma_{\Gamma}(P)$ be countable. If there exists a bounded uniformly continuous solution $u$ to (1), then it is almost periodic. Moreover, the following part of the Fourier series of $u$ :

$$
\sum b_{\lambda} e^{i \lambda t}, \quad b_{\lambda}=\lim _{T \rightarrow \infty} \frac{1}{2 T} \int_{-T}^{T} e^{-i \lambda \xi} u(\xi) d \xi
$$

where $e^{i \lambda} \in \sigma(f)$, is again the Fourier series of another almost periodic solution to (1). 
Proof. The assertion that $u$ is almost periodic is standard in view of (11) (see e.g. [L-Z], [A-B], [Ba]). It may be noted that in case $u$ is almost periodic, the spectral decomposition can be carried out in the function space $\operatorname{AP}(\mathbf{X})$ instead of the larger space $\mathrm{BUC}(\mathbb{R}, \mathbf{X})$. Hence, we can decompose the solution $u$ into the sum of two almost periodic solutions with spectral properties described in Theorem 3.3. Using the definition of Fourier series of almost periodic functions we arrive at the next assertion of the corollary.

The following corollary will show the advantage of Theorem 3.5 which allows us to take into account the structure of $\operatorname{sp}(f)$ rather than that of $\sigma(f)$. To this end, we introduce the following terminology. A set $S$ of reals is said to have a finite integer basis if there is a finite subset $T \subset S$ such that any element $s \in S$ can be represented in the form $s=n_{1} b_{1}+\ldots+n_{m} b_{m}$, where $n_{j} \in \mathbb{Z}$, and $b_{j} \in T$ for $j=1, \ldots, m$. If $f$ is quasi-periodic, i.e., of the form $f(t)=F(t, \ldots, t), t \in \mathbb{R}$, where $F\left(t_{1}, \ldots, t_{n}\right)$ is an $\mathbf{X}$-valued continuous function of $n$ variables which is periodic in each variable, and the set of its Fourier-Bohr exponents is discrete (it coincides with $\operatorname{sp}(f)$ in this case), then the spectrum $\operatorname{sp}(f)$ has a finite integer basis (see [L-Z, p. 48]). Conversely, if $f$ is almost periodic and $\operatorname{sp}(f)$ has a finite integer basis, then $f$ is quasi-periodic. We refer the reader to [L-Z, pp. 42-48] for more information on the relation between quasi-periodicity, spectrum and Fourier-Bohr exponents of almost periodic functions.

COROLlary 4.4. Let all assumptions of the second assertion of Theorem 3.5 be satisfied. Moreover, assume that $\mathbf{X}$ does not contain $c_{0}$. If $\operatorname{sp}(f)$ has a finite integer basis, then (15) has a quasi-periodic mild solution w with $\operatorname{sp}(w)=\operatorname{sp}(f)$.

Proof. Under the assumptions of the corollary the spectrum $\operatorname{sp}(w)$ of the solution $w$, as described in Theorem 3.5, is in particular countable. Hence $w$ is almost periodic. Since $\operatorname{sp}(w)=\operatorname{sp}(f), \operatorname{sp}(w)$ has an a finite integer basis. Thus $w$ is quasi-periodic.

Below we consider some particular cases:

EXAMPLE 1 (Periodic solutions). If $\sigma(f)=\{1\}$ we are actually concerned with the existence of periodic solutions. Hence, Corollary 4.2 extends the classical result by Massera to a large class of evolution equations which have 1 as an isolated point of $\sigma_{\Gamma}(P)$. Moreover, Corollary 4.3 provides a way to approximate the periodic solution. (See $[\mathrm{N}-\mathrm{M}-\mathrm{M}-\mathrm{S}]$ for the case where the monodromy operator $P$ is compact.)

EXAMPle 2 (Anti-periodic solutions). A (continuous) function $f$ is defined to be anti-periodic if $f(t+\omega)=-f(t)$ for all $t \in \mathbb{R}$ and fixed $\omega>0$. Thus, $f$ is $2-\omega$-periodic. It is known that the space of anti-periodic functions $f$ with antiperiod $\omega$, which is denoted by $\mathcal{A P}(\omega)$, is a subspace of 
$\operatorname{BUC}(\mathbb{R}, \mathbf{X})$ with

$$
\operatorname{sp}(f) \subset\left\{\frac{2 k+1}{\omega}: k \in \mathbb{Z}\right\} .
$$

Without loss of generality we can assume that $\omega=1$. Obviously, $\sigma(f)=$ $\{-1\}$ for all $f \in \mathcal{A P}(\omega)$ (for more information on anti-periodic solutions see $[\mathrm{A}-\mathrm{P}],[\mathrm{Nak}],[\mathrm{O}],[\mathrm{V}-\mathrm{S}])$. In this case the spectral separation condition (Definition 4.1) is nothing but the condition that $\{-1\}$ is an isolated point of $\sigma_{\Gamma}(P)$. Hence, we have extended Massera's result to anti-periodic solutions.

EXAMPLE 3 . Let $u$ be a bounded uniformly continuous solution to (1) with $f$ 2-periodic. Define

$$
F(t)=\frac{f(t)-f(t+1)}{2}, \quad G(t)=\frac{f(t)+f(t+1)}{2}, \quad \forall t \in \mathbb{R} .
$$

Then it is seen that $F$ is 1 -anti-periodic and $G$ is 1 -periodic. Applying Theorem 3.3 we see that there exist two solutions to (1), namely the two components of $u$ which are 1-anti-periodic and 1-periodic with forcing terms $F, G$, respectively. In particular, the sum of these solutions is a 2-periodic solution of (1) with forcing term $f$.

EXAmple 4. Let $A$ be a sectorial operator in a Banach space $\mathbf{X}$. As usual, for $\alpha \geq 0$ we use the following notations:

$$
\mathbf{X}^{\alpha}:=D\left(A_{1}^{\alpha}\right), \quad \text { with the graph norm }\|x\|_{\alpha}:=\left\|A_{1}^{\alpha} x\right\|, x \in \mathbf{X}^{\alpha},
$$

where $A_{1}=A+a I$ with $a$ chosen so that $\operatorname{Re} \lambda>0$ for all $\lambda \in \sigma\left(A_{1}\right)$ and $A_{1}^{\alpha}$ is the fractional power of $A_{1}$ (see e.g. [He, pp. 24-29]). With these notations $\left(\mathbf{X}^{\alpha},\|\cdot\|_{\alpha}\right)$ is a Banach space. Suppose that $t \mapsto B(t) \in L\left(\mathbf{X}^{\alpha}, \mathbf{X}\right)$ is Hölder continuous and 1-periodic. Then, as shown in [He, Thm. 7.1.3], the equation

$$
\frac{d x}{d t}=(-A+B(t)) x
$$

generates a 1-periodic strongly continuous evolutionary process $(U(t, s))_{t \geq s}$. If, furthermore, $A$ has compact resolvent, then the monodromy operator $P$ of the process is compact. Hence, for every almost periodic function $f$ the sets $\sigma(f)$ and $\sigma_{\Gamma}(P)$ always satisfy the spectral separation condition. In [N-M] we have shown that if $\sigma_{\Gamma}(P) \cap \sigma(f)=\emptyset$, then there is a unique almost periodic solution $x_{f}$ to the inhomogeneous equation

$$
\frac{d x}{d t}=(-A+B(t)) x+f(t)
$$

with the property that $\sigma\left(x_{f}\right) \subset \sigma(f)$. Now suppose that $\sigma_{\Gamma}(P) \cap \sigma(f) \neq \emptyset$. By Corollary 4.2, if $u$ is any bounded solution (the uniform continuity follows from the boundedness of such a solution to (19)), then there exists an almost periodic solution $w$ such that $\sigma(w)=\sigma(f)$. We refer the reader to [He] 
and $[\mathrm{Paz}]$ for examples from parabolic differential equations which can be included into the abstract equation (19).

EXAMPLE 5. Consider the heat equation in materials

$$
\left\{\begin{array}{l}
v_{t}(t, x)=\Delta v(t, x)+f(t, x), \quad t \in \mathbb{R}, x \in \Omega, \\
v(t, x)=0, \quad t \in \mathbb{R}, x \in \partial \Omega
\end{array}\right.
$$

where $\Omega \subset \mathbb{R}^{n}$ denotes a bounded domain with smooth boundary $\partial \Omega$. Let $\mathbf{X}=L^{2}(\Omega), A=\Delta$ with $D(A)=W^{2,2}(\Omega) \cap W_{0}^{1,2}(\Omega)$. Then $A$ is selfadjoint and negative definite (see e.g. [Paz]). Hence $\sigma(A) \subset(-\infty, 0)$. In particular $\sigma_{i}(A)=\emptyset$. Now $(20)$ becomes

$$
\frac{d v}{d t}=A v+f
$$

We assume further that $f(t, x)=a(t) g(x)$ where $a$ is a bounded uniformly continuous real function with $\operatorname{sp}(a)=\mathbb{Z} \cup \pi \mathbb{Z}, g \in L^{2}(\Omega), g \neq 0$. It may be seen that $\sigma(f)=S^{1}$ and $\operatorname{sp}(f)$ has a finite integer basis. Hence, Theorem 3.3 does not give any information on the existence of a solution $w$ with specific spectral properties. However, in this case Theorem 3.5 applies.

Acknowledgements. The paper was written while N.V.M. was being a fellow of the Japan Society for the Promotion of Science. The generous support of the Society is gratefully acknowledged. The authors wish to thank the referee for several remarks concerning the presentation of the paper. The second author (N.V.M.) would like to dedicate this work to Professor Peter P. Zabreiko on the occasion of his 60th birthday.

\section{References}

[A-P] S. Aizicovici and N. Pavel, Anti-periodic solutions to a class of nonlinear differential equations in Hilbert space, J. Funct. Anal. 99 (1991), 387-408.

[A-B] W. Arendt and C. J. K. Batty, Almost periodic solutions of first and second order Cauchy problems, J. Differential Equations 137 (1997), 363-383.

[A-S] W. Arendt and S. Schweiker, Discrete spectrum and almost periodicity, Taiwanese J. Math. 3 (1999), 475-490.

[A-M] B. Aulbach and Nguyen Van Minh, Nonlinear semigroups and existence, stability of semilinear nonautonomous evolution equations, Abstract Appl. Anal. 1 (1996), 351-380.

[Ba] B. Basit, Harmonic analysis and asymptotic behavior of solutions to the abstract Cauchy problem, Semigroup Forum 54 (1997), 58-74.

[B-H-R] C. J. K. Batty, W. Hutter and F. Räbiger, Almost periodicity of mild solutions of inhomogeneous periodic Cauchy problems, J. Differential Equations 156 (1999), 309-327.

[C-H] S. N. Chow and J. K. Hale, Strongly limit-compact maps, Funkc. Ekvac. 17 (1974), 31-38. 
[D-M] D. Daners and P. K. Medina, Abstract Evolution Equations, Periodic Problems and Applications, Pitman Res. Notes in Math. Ser. 279, Longman, New York, 1992.

[D] E. B. Davies, One-Parameter Semigroups, Academic Press, London, 1980.

[E-Na] K.-J. Engel and R. Nagel, One-Parameter Semigroups for Linear Evolution Equations, Springer, 2000.

[F] A. M. Fink, Almost Periodic Differential Equations, Lecture Notes in Math. 377, Springer, Berlin, 1974.

[He] D. Henry, Geometric Theory of Semilinear Parabolic Equations, Lecture Notes in Math. 840, Springer, Berlin, 1981.

[J] R. Johnson, A linear, almost periodic equation with an almost automorphic solution, Proc. Amer. Math. Soc. 82 (1981), 199-205.

[Ka] Y. Katznelson, An Introduction to Harmonic Analysis, Dover Publ., New York, 1968.

[L-Z] B. M. Levitan and V. V. Zhikov, Almost Periodic Functions and Differential Equations, Moscow Univ. Publ. House, 1978 (in Russian); English transl. by Cambridge Univ. Press, 1982.

[Ma] J. L. Massera, The existence of periodic solutions of systems of differential equations, Duke Math. J. 17 (1950), 457-475.

[M-N-M] S. Murakami, T. Naito and Nguyen Van Minh, Evolution semigroups and sums of commuting operators: a new approach to the admissibility theory of function spaces, J. Differential Equations 164 (2000), 240-285.

[N-M] T. Naito and Nguyen Van Minh, Evolution semigroups and spectral criteria for almost periodic solutions of periodic evolution equations, ibid. 152 (1999), 358-376.

[N-M-M-S] T. Naito, Nguyen Van Minh, R. Miyazaki and J. S. Shin, A decomposition theorem for bounded solutions and the existence of periodic solutions to periodic differential equations, ibid. 160 (2000), 263-282.

[Na] R. Nagel (ed.), One-Parameter Semigroups of Positive Operators, Lecture Notes in Math. 1184, Springer, Heidelberg, 1984.

[Nak] M. Nakao, Existence of an anti-periodic solution for the quasilinear wave equation with viscosity, J. Math. Anal. Appl. 204 (1996), 754-764.

[Ne] J. M. A. M. van Neerven, The Asymptotic Behavior of Semigroups of Linear Operators, Birkhäuser, Basel, 1996.

[O] H. Okochi, On the existence of anti-periodic solutions to a nonlinear evolution equation associated with odd subdifferential operators, J. Funct. Anal. 91 (1990), 246-258.

[Paz] A. Pazy, Semigroups of Linear Operators and Applications to Partial Differential Equations, Appl. Math. Sci. 44, Springer, Berlin, 1983.

[Pr] J. Prüss, Evolutionary Integral Equations and Applications, Birkhäuser, Basel, 1993.

[R-V] W. M. Ruess and Q. P. Vu, Asymptotically almost periodic solutions of evolution equations in Banach spaces, J. Differential Equations 122 (1995), 282301.

[S-Y] W. Shen and Y. Yi, On minimal sets of scalar parabolic equations with skewproduct structures, Trans. Amer. Math. Soc. 347 (1995), 4413-4431.

[S-N] J. S. Shin and T. Naito, Semi-Fredholm operators and periodic solutions for linear functional differential equations, J. Differential Equations 153 (1999), 407-441. 
[V] Q. P. Vu, Stability and almost periodicity of trajectories of periodic processes, ibid. 115 (1995), 402-415.

[V-S] Q. P. Vu and E. Schüler, The operator equation $A X-X B=C$, stability and asymptotic behaviour of differential equations, ibid. 145 (1998), 394-419.

[Ya] M. Yamaguchi, Nonexistence of bounded solutions of one-dimensional wave equations with quasiperiodic forcing terms, ibid. 127 (1996), 484-497.

Department of Mathematics

University of Electro-Communications

Chofu, Tokyo 182-8585, Japan

E-mail: naito@matha.e-one.uec.ac.jp

Department of Mathematics

Korea University

Kodaira, Tokyo 187-8560, Japan

E-mail: shinjs@tech.korea-u.ac.jp
Permanent address:

Department of Mathematics

Hanoi University of Science

334 Nguyen Trai, Hanoi, Vietnam

Current address:

Department of Mathematics

University of Electro-Communications

Chofu, Tokyo 182-8585, Japan

E-mail: minh@matha.e-one.uec.ac.jp

Received March 23, 1999

Revised version November 17, 2000 